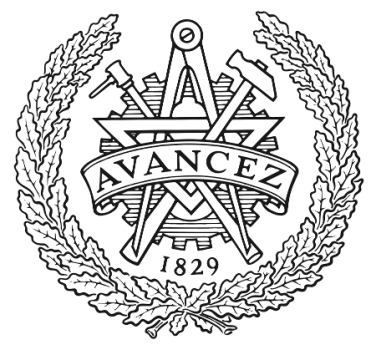

CHALMERS

UNIVERSITY OF TECHNOLOGY

\title{
Simple Boundary Condition for Canonical EBG Surface: PMC-Backed Uniaxial Medium
}

Downloaded from: https://research.chalmers.se, 2023-04-26 07:31 UTC

Citation for the original published paper (version of record):

Bosiljevac, M., Sipus, Z., Kildal, P. et al (2016). Simple Boundary Condition for Canonical EBG

Surface: PMC-Backed Uniaxial Medium. IEEE Transactions on Antennas and Propagation, 64(2):

785-U550. http://dx.doi.org/10.1109/tap.2015.2506721

N.B. When citing this work, cite the original published paper.

C2016 IEEE. Personal use of this material is permitted.

However, permission to reprint/republish this material for advertising or promotional purposes 


\section{Simple Boundary Condition for Canonical EBG surface: PMC-backed Uniaxial Medium}

\author{
Marko Bosiljevac, Zvonimir Sipus, Per-Simon Kildal \\ and Angelo Freni
}

\begin{abstract}
A simple-to-use replacement model for isotropic electromagnetic bandgap surfaces such as mushroom surfaces is investigated. Properties of electromagnetic bandgap surfaces strongly depend on the incidence angle of the incoming plane wave. The suggested model takes this behavior into account and actually represents the ideal electromagnetic bandgap surface. The model is based on uniaxial representation of a thin DB layer backed by a PMC plate. We investigate how this model behaves in comparison with a realistic mushroom surface, and when it can be applied. The results show that the proposed model can be used for both far field calculations and antenna coupling evaluation.
\end{abstract}

Index Terms-Periodic surfaces, electromagnetic scattering, EBG surfaces, anisotropic surfaces, canonical boundary conditions

\section{INTRODUCTION}

$\mathrm{I}$ deal boundary conditions are widely used in most electromagnetic solvers to approximate the behavior of actual surfaces or materials. For example, it is customary to replace metal conductors with Perfect Electric Conductors (PEC), thus greatly simplifying the computation. Perfect Magnetic Conductor (PMC) boundary condition is also often used in the first stage of electromagnetic simulations, even though magnetic conductors at microwave frequencies do not exist in nature. The reason is that some artificial surfaces exhibit magnetic conducting properties in certain frequency bands. Such surfaces normally have a very complex periodic structure, whose analysis requires very time-consuming full wave techniques, especially if the surfaces are a part of a larger electromagnetic structure. In such cases, PMC offers an easy way to simplify the analysis and still provides practically useful results [1]-[3].

The PEC and PMC are defined by the condition that tangential electric field and tangential magnetic field are zero at the boundary, respectively. By combining PEC strips and PMC strips parallel to each other, we can obtain other quite

Manuscript submitted July $17^{\text {th }} 2015$; revised November $25^{\text {th }} 2015$; accepted November $29^{\text {th }} 2015$. This work was supported in part by the Swedish Foundation for Strategic Research (SSF) via the CHARMANT antenna systems research center at Chalmers, and by The Swedish Governmental Agency for Innovation Systems (VINNOVA) within the VINN Excellence Center Chase.

M. Bosiljevac and Z. Sipus are with Faculty of Electrical Engineering and Computing, University of Zagreb, Croatia (email: marko.bosiljevac@fer.hr, zvonimir.sipus@fer.hr).

P.-S. Kildal is with Chalmers University of Technology (Chalmers), Gothenburg, Sweden (email: per-simon.kildal@chalmers.se).

A. Freni is with University of Florence, Florence, Italy (e-mail: angelo.freni@unifi.it). common ideal surfaces, i.e. the so-called soft and hard surfaces [3]-[5]. The former is observed when waves are propagating transverse to the $\mathrm{PEC} / \mathrm{PMC}$ strips, and the latter when the waves propagate along them. Ideal simple surfaces of these kinds can be referred to as canonical surfaces [1]. Lately, several new boundary conditions with interesting properties such as DB, D'B', SHDB have been presented [6] and this list will, for sure, expand in the following years. However, the main practical issue regarding these boundary conditions, namely their realization, still remains mostly unanswered. For this reason, we are considering different ways of exploiting these types of boundary conditions and in particular DB boundary properties.

Many artificial surface types, apart from the ideal ones mentioned above, have appeared during the recent years, and they are commonly used in various applications. In particular, artificial surfaces that exhibit electromagnetic bandgap (EBG) properties, such as mushroom structures, have proven very useful [3], [7]. They are successfully used for both antenna coupling reduction and for achieving artificial magnetic ground planes for low profile antennas. EBG surfaces incorporate many fine details in their design and consequently the analysis of devices which contain them is often quite complex, and there is a need for simpler representation of the EBG surface. This can be beneficial in initial designs to achieve the desired goal, i.e. to determine which improvements in the antenna system are possible to obtain by using artificial surfaces.

The characteristics of EBG surfaces change significantly on the angle of incoming wave. For grazing incidence they behave basically as isotropic soft surfaces stopping any kind of wave propagation along the surface. For normal incidence, they behave as Artificial Magnetic Conductors (AMC). This behavior cannot be captured by simple boundary conditions like PEC or PMC, but solutions exist if only certain aspects of the EBG surface need to be modeled. Boundary conditions that describe ideal isotropic soft surface are the DB boundary conditions introduced by Rumsey in 1959 [8] and recently investigated by Lindell and Sihvola in [6], [9], [10]. Here, DB refers to a surface for which the normal components of both $D$ and $\mathrm{B}$ vectors are defined to be zero, thus forcing the decay of both TE and TM polarized waves along the surface when propagating away from a source located at the surface. Such defined DB boundary conditions can also be written as

$$
E_{n}=0, \quad H_{n}=0
$$

in all practical cases when the medium on one side of the boundary is isotropic (here index $n$ denotes normal field components). Although very simple and easy to implement, these boundary conditions are very powerful and they are a good starting point in the quest to describe the ideal EBG surface, but they cannot be used directly since they lack definition for normal incidence [10] [11].

An attempt to modify DB conditions to approach the ideal EBG surface conditions were the PMC amended DB boundary conditions [11], defined as 


$$
H_{n}-j H_{l}=0, E_{n}=0,
$$

where index $n$ denotes the normal component of the field (with respect to the boundary surface), and $l$ denotes its longitudinal component in the direction of wave propagation along the boundary surface. They correct the normal incidence anomaly present in the definition of DB conditions and can very well describe practical EBG surfaces. However, the problem of the amended DB conditions lies in the fact that the direction of the wave impinging on the surface needs to be known, which is not suitable for implementation in general EM solvers. Consequently, we have taken a new approach based on uniaxial representation of the original DB boundary [9], and we have extended this in a physical way in order to describe correctly the EBG surface also for normal incidence. This approach is a more practical way of establishing a canonical EBG boundary, and thanks to its simplicity it has potential for application in general EM codes for arbitrary geometries and sources. Comparison of basic properties of all considered surfaces is given in Table 1 .

TABLE 1 COMPARISON OF BASIC PROPERTIES OF PRACTICAL EBG SURFACE, DB SURFACE, AND PMC-BACKED DB SURFACE.

\begin{tabular}{|c|c|c|c|}
\hline & \multirow{2}{*}{$\begin{array}{l}\text { Normal } \\
\text { incidence }\end{array}$} & \multicolumn{2}{|c|}{ Grazing incidence } \\
\hline & & $\begin{array}{l}\mathrm{TE} \\
\text { polarization }\end{array}$ & $\begin{array}{l}\text { TM } \\
\text { polarization }\end{array}$ \\
\hline $\begin{array}{l}\text { Practical } \\
\text { EBG surface }\end{array}$ & $\begin{array}{l}\text { approx. } \\
\text { PMC }\end{array}$ & PEC & PMC \\
\hline DB surface & not defined & PEC & PMC \\
\hline $\begin{array}{l}\text { PMC-backed } \\
\text { DB surface }\end{array}$ & PMC & PEC & PMC \\
\hline
\end{tabular}

\section{PMC-BACKed DB UNIAXIAL MEDIUM}

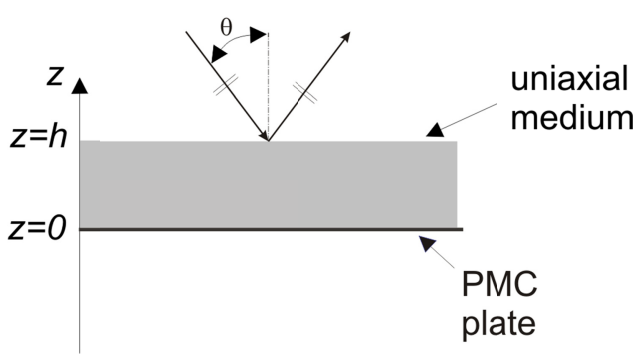

Fig.1. Geometry of the uniaxial medium representing DB slab backed with a PMC plane

The implementation of DB boundary conditions that we shall consider is a uniaxial anisotropic slab shown in Fig. 1 characterized by tensors

$$
\overline{\bar{\varepsilon}}=\varepsilon_{0}\left[\begin{array}{ccc}
\varepsilon_{x} & 0 & 0 \\
0 & \varepsilon_{x} & 0 \\
0 & 0 & \varepsilon_{z}
\end{array}\right], \overline{\bar{\mu}}=\mu_{0}\left[\begin{array}{ccc}
\mu_{x} & 0 & 0 \\
0 & \mu_{x} & 0 \\
0 & 0 & \mu_{z}
\end{array}\right] .
$$

It is worth noting that by forcing $\varepsilon_{z}$ and $\mu_{z}$ to zero we obtain the DB boundary conditions at the air-medium interface [9], because $D_{z}=\varepsilon_{0} \varepsilon_{z} E_{z}$ and $B_{z}=\mu_{0} \mu_{z} H_{z}$.

Using similar procedure as in [12], [13] we obtain the following differential equations for the $E_{\mathrm{z}}$ and $H_{\mathrm{z}}$ components of the electromagnetic field:

$$
\begin{aligned}
& \frac{\partial^{2} E_{z}}{\partial x^{2}}+\frac{\partial^{2} E_{z}}{\partial y^{2}}+\frac{\varepsilon_{z}}{\varepsilon_{x}} \frac{\partial^{2} E_{z}}{\partial z^{2}}+\mu_{x} \varepsilon_{z} k_{0}^{2} E_{z}=0 \\
& \frac{\partial^{2} H_{z}}{\partial x^{2}}+\frac{\partial^{2} H_{z}}{\partial y^{2}}+\frac{\mu_{z}}{\mu_{x}} \frac{\partial^{2} H_{z}}{\partial z^{2}}+\varepsilon_{x} \mu_{z} k_{0}^{2} H_{z}=0
\end{aligned},
$$

where $k_{0}^{2}=\omega^{2} \varepsilon_{0} \mu_{0}$. By assuming plane wave propagation in a standard form $e^{-j k_{x} x} e^{-j k_{y} y} e^{-j k_{z} z}$ the following dispersion relation is obtained for the $E_{z}$ field (TM case)

$$
\left(k_{z}^{T M}\right)^{2}=\varepsilon_{x} \mu_{x} k_{0}^{2}-\frac{\varepsilon_{x}}{\varepsilon_{z}} \beta^{2},
$$

while for the $H_{z}$ field (TE case) it is

$$
\left(k_{z}^{T E}\right)^{2}=\varepsilon_{x} \mu_{x} k_{0}^{2}-\frac{\mu_{x}}{\mu_{z}} \beta^{2},
$$

where $\beta^{2}=k_{x}^{2}+k_{y}^{2}$.

Next, we apply a modification of the structure in which the thin uniaxial slab is backed with a PMC plate. By making the structure thin and letting $\varepsilon_{z}$ and $\mu_{z}$ approach to zero, we will show that we obtain boundary conditions that correspond to practical EBGs.

The reflection coefficients for the latter geometry (shown in Fig. 1), that we will call PMC-backed uniaxial slab, are:

$$
\begin{gathered}
\Gamma_{T E}=\frac{k_{z}-j k_{z}^{T E} \tan \left(k_{z}^{T E} h\right)}{k_{z}+j k_{z}^{T E} \tan \left(k_{z}^{T E} h\right)}, \\
\Gamma_{T M}=\frac{k_{z}+j k_{z}^{T M} \cot \left(k_{z}^{T M} h\right)}{k_{z}-j k_{z}^{T M} \cot \left(k_{z}^{T M} h\right)} .
\end{gathered}
$$

These coefficients depend on the slab thickness and on $\varepsilon_{z}$ and $\mu_{z}$ components of uniaxial medium. This will result in a possibility to adjust the phase behavior of the PMC backed uniaxial slab in order to match the desired characteristics of the practical EBG structure.

\section{Practical Case: Dipole over Planar EBG}

\section{A. Far-field results}

As an example of EBG structure we shall consider the mushroom structure introduced in [7] and shown in Figure 2.a, with dimensions: width of the square patches $w=2.25 \mathrm{~mm}$, period in both $x$ and $y$ directions $P=2.4 \mathrm{~mm}$, thickness and permittivity of the dielectric slab $t=1.6 \mathrm{~mm}$, relative permittivity $\varepsilon_{r}=2.2$, and vias diameter $d=0.36 \mathrm{~mm}$. Fullwave solutions for the mushroom surface including all 
geometrical details have been obtained by using CST Microwave Studio [14] and 3DAMxLAD (3D Antennas Modeller eXtended for Large Array Design) [15], [16] numerical codes. CST MS was also used to simulate the canonical PMC-backed uniaxial medium itself (shown in Fig. 2.b). Figs. 2.a and 2.b also show a horizontal dipole, which will be used to compare near and far-field effects between the realistic mushroom structure and the proposed model.

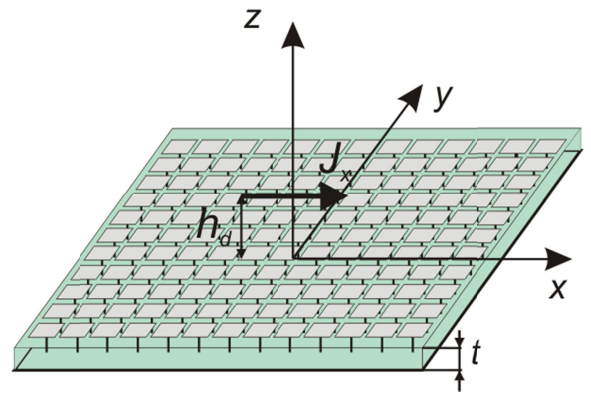

(a)

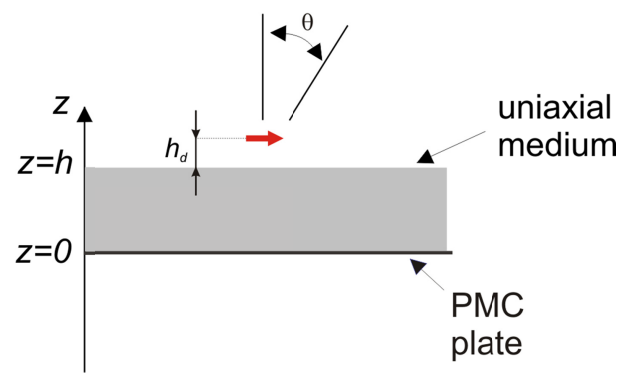

(b)

Fig. 2. (a) Mushroom structure geometry excited by a short horizontal dipole (b) equivalent PMC backed uniaxial medium EBG model geometry excited by a short horizontal dipole.

Characteristics of practical EBG structures, as mentioned before, are highly erratic if we consider a wide frequency range, however, for the purpose of modeling we can concentrate on the behavior that is most dominant and is visible approximately in the center of the bandgap (the measured bandgap is between 11.5 and $16.6 \mathrm{GHz}$ [7]). We refer here to the part of the bandgap or frequency where the phase characteristics of practical EBG are closest to the ideal ones. For this reason Fig. 3 shows the phase of the reflection coefficient of the EBG structure versus the angle of incidence of the impinging plane wave. Almost ideal behavior (i.e. when EBG acts as a PMC for normal incidence) is present around 14 $\mathrm{GHz}$, while if we move towards the two ends of the bandgap (i.e. $12 \mathrm{GHz}$ and $16 \mathrm{GHz}$ ) phase characteristics change significantly.

Looking at Fig. 3 it is clear that the modeling frequency should be set to $14 \mathrm{GHz}$, and the parameters $h, \varepsilon_{z}$ and $\mu_{z}$ of the uniaxial slab have to be chosen in a way to match the phase characteristic of the actual EBG. The ideal phase of reflection coefficient for TM case is $0^{\circ}$ (PMC) for all incidence angles. To obtain this, the thickness of the uniaxial slab $h$ should be electrically small (typically $\lambda_{0} / 100$ or even $\lambda_{0} / 1000$ ). In this case the value of the $z$-component of permittivity is not critical since the boundary condition for the normal component of $E$ field at PMC surface (i.e., $E_{\mathrm{z}}=0$ ), due to small thickness of the slab, is practically directly translated to the uniaxial medium/air interface $(z=h)$. Therefore, for sake of simplicity, we will use $\varepsilon_{z}=1.0$ in all considered cases, and also $\varepsilon_{x}=\mu_{x}=$ 1.0 since this choice avoids unnecessary propagation of waves along the surface.

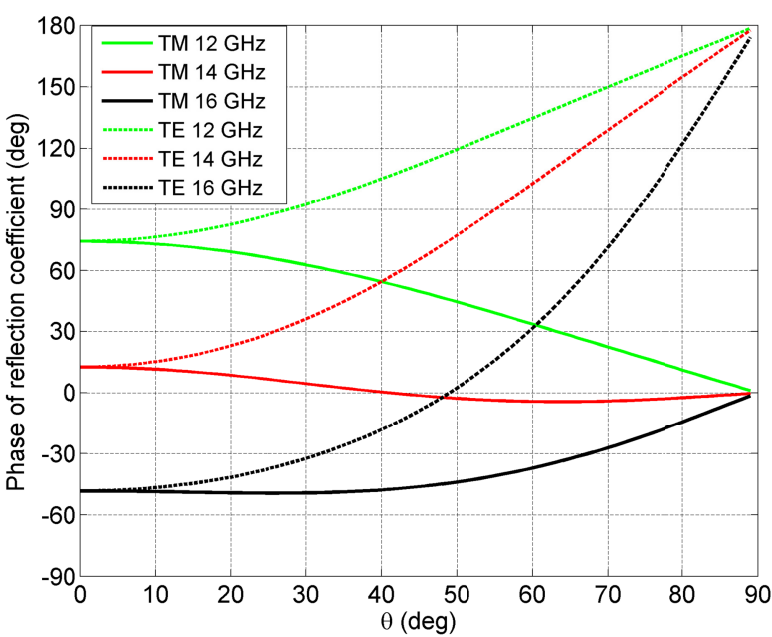

Fig. 3. Reflection coefficient phase for TE and TM cases, computed for the practical mushroom structure by considering it as infinite double periodic structure.

The ratio $\mu_{z} / h$, however, is a critical parameter since it governs the slope of the TE polarization reflection coefficient argument. In the ideal case the TE phase goes from 0 to $180^{\circ}$ when scanning the incident angle $\theta$. This is seen in the realistic TE case at $14 \mathrm{GHz}$ shown in Fig. 3 computed for an infinite double periodic EBG structure. To match this exact slope we force the phase of the reflection coefficient in (7) to be equal to the reflection coefficient phase value computed in Fig. 3. Since the matching can be obtained only for a single angle of incidence, in order to reduce the average error, we choose an angle in the center of the scan area (specifically $\theta=45^{\circ}$ ). With this choice, the value of the ratio that ensures the best matching for the EBG under analysis is:

$$
\frac{\mu_{z}}{h}=\frac{8.9}{\lambda}
$$

Additional to this specific EBG, we have considered also different EBG designs found in scientific literature (e.g. in [3]), and the ratio (9) fits these designs as well. To verify the accuracy of the approximation we first compare the phase values of the reflection coefficients for both TE and TM cases when calculated by using CST Microwave Studio for both the canonical EBG model (i.e. of PMC-backed uniaxial medium) and the realistic mushroom surface. The result is shown in Fig. 4 for the working frequency of $14 \mathrm{GHz}$ at which the considered mushroom surface acts as a PMC for normal incidence. 
The PMC-backed uniaxial model is further verified by considering a short dipole (10 mm long) placed horizontally $0.5 \mathrm{~mm}$ from the EBG structure, as shown in Fig. 2. This configuration allows us to check the accuracy of the radiated field and the possibility to model near-field effects.

In the case of the PMC-backed uniaxial model the field radiation pattern can be obtained very simply by summing the contributions directly radiated from the dipole and those reflected from the EBG surface, as follows:

$$
E_{H-\text { plane }}=C \frac{e^{-j k R}}{R} \cdot\left(1+\Gamma_{T E}(\theta) \cdot e^{-j 2 k_{0} h_{d} \cos \theta}\right)
$$

for the TE case (H plane), and

$$
E_{E-\text { plane }}=C \frac{e^{-j k R}}{R} \cos \theta \cdot\left(1-\Gamma_{T M}(\theta) \cdot e^{-j 2 k_{0} h_{d} \cos \theta}\right)
$$

for the TM case (E plane), with $C=-j \eta k / 4 \pi$.

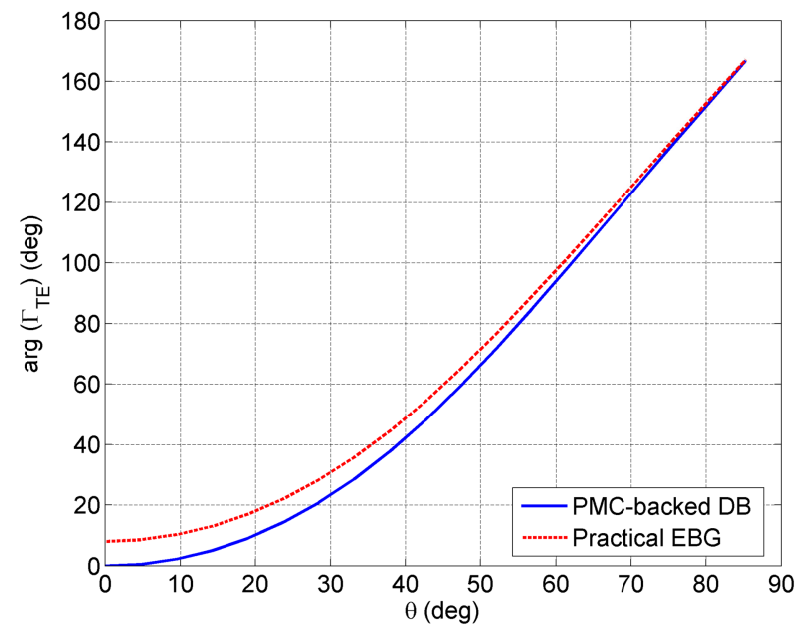

(a)

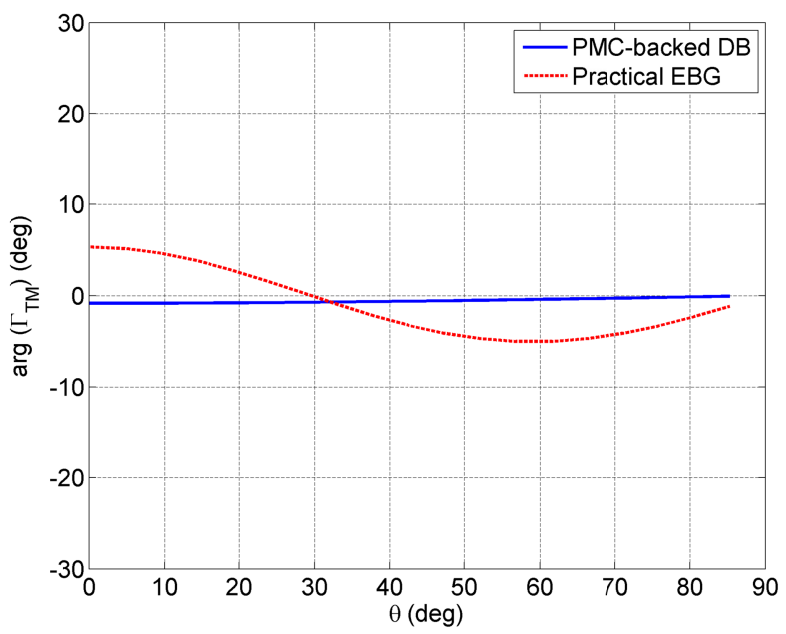

(b)

Fig. 4. Reflection coefficient phase, computed for the PMC backed uniaxial model $(h=\lambda / 1000)$ and for the actual mushroom surface at $14 \mathrm{GHz}$ versus the incidence angle of the impinging plane wave; (a) TE case, (b) TM case.
The patterns in both E-plane and H-plane are shown in Fig. 5. It shows how well the PMC-backed uniaxial medium model matches with the results obtained by the general EM solver (CST Microwave Studio) for the realistic case of a short dipole above the mushroom surface of finite dimensions ( $5 \lambda \mathrm{x}$ $5 \lambda$ structures were simulated). On the other side, the PMC model completely failed in prediction of radiation pattern in the H-plane. Therefore, we can say that the proposed model can be easily used as an ideal replacement for an EBG surface working in the middle of the frequency bandgap. This speeds up the process of making the first design of some EM structure that contains EBG layers.

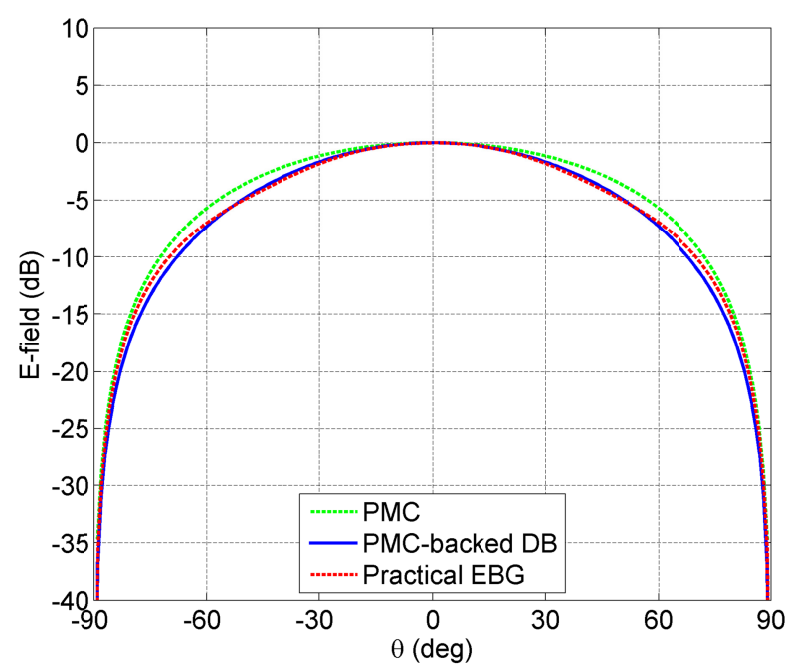

(a)

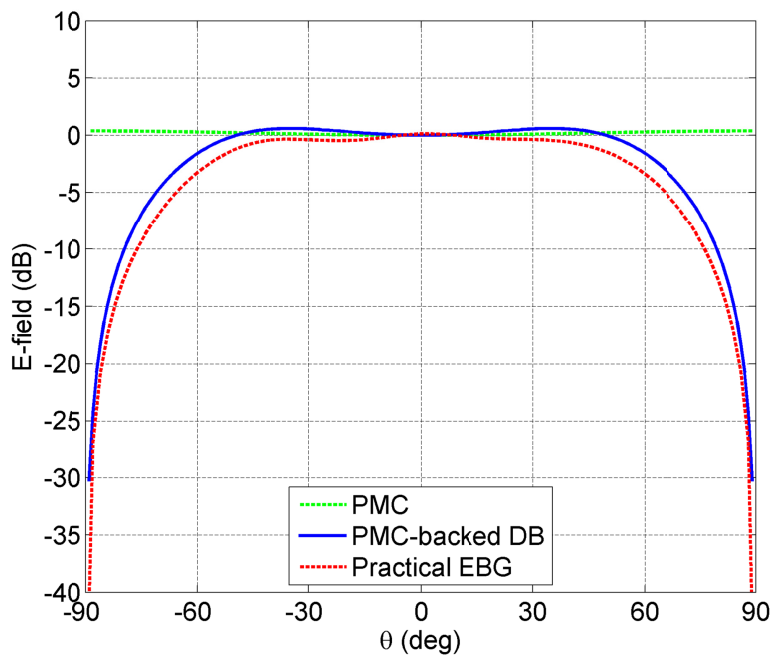

(b)

Fig. 5. Radiation pattern of a horizontal short dipole placed $0.5 \mathrm{~mm}$ above a realistic mushroom surface; (a) $E$ plane, (b) $H$ plane.

\section{B. Near-field results}

Since the radiation pattern results have a good matching between the canonical model and the real structure, the natural question that arises is if the canonical model can also be used for prediction of near-field parameters. Thus, we have calculated the $S_{11}$ parameter versus frequency for a horizontal dipole placed above the EBG structure (the dimensions of the 
structure are the same as in the previous examples). The EBG structure was analyzed using the full-wave 3DAMxLAD code [15], [16], while for analyzing dipoles above a PMC-backed uniaxial structure and above a PMC plane a moment method code was developed based on the approach given in [17]. It is worth noting that Fig. 6 shows a shift in matching frequency between our model and the realistic structure. However, a shift between the PMC model and the realistic structure is also present. Therefore, the proposed canonical model is of the same order of accuracy as the PMC model when calculating the input reflection coefficient (in average it even shows a better agreement).

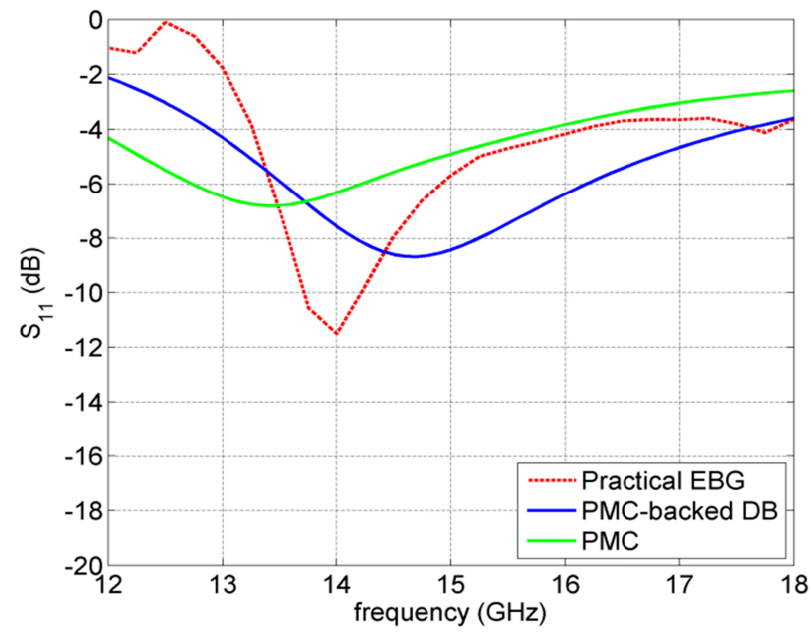

Fig. 6. $S_{11}$ comparison for small dipole above different structures; actual mushroom structure, ideal PMC and PMC-backed uniaxial model.

Much better agreement between the PMC-backed uniaxial model and the practical EBG surface can be obtained when calculating coupling between two dipoles placed above the considered EBG surface. As an example Fig. 7 shows the $S_{21}$ parameter calculated for two dipoles both in the $\mathrm{H}-$ and $\mathrm{E}$ plane (to nullify the influence of the input impedance mismatch, the $\left|S_{21}\right|^{2}$ values are normalized with $\left(1-\left|S_{11}\right|^{2}\right)^{2}$ ). While in the E-plane (Fig. 7.b) the difference between models is not large (in that case EBG surface acts as a PMC plane), in the H-plane (Fig. 7.a) there is a drastic difference between two canonical models. It can be noted that the PMC-backed uniaxial model predicts very well the magnitude and the decaying tendency of the $S_{2 I}$ parameter, while the PMC model completely failed in prediction of $S_{2 I}$ parameter. This is due to the fact that for TE polarization the EBG surface acts as a PEC surface for grazing incidence.

\section{CONCLUSION}

Canonical behavior of electromagnetic bandgap (EBG) surfaces is modeled using PMC backed uniaxial DB model. The idea is to use this canonical model to replace generic EBG surfaces in general electromagnetic solvers and obtain first hand results without the need for accurate modeling of EBG with its many fine details. The ideal properties of EBGs (at the desired frequency) are reasonably well reproduced by the PMC backed DB model. This is verified through the comparison of the proposed model results with the full-wave simulation results for the realistic EBG surface. The results show that the far-field is well reproduced while the near-field results have to be taken with precautions. Namely, the $S_{I I}$ has an offset in the matching frequency when compared to the realistic EBG, while on the other hand the coupling $S_{21}$ is predicted very well. Taking these precautions into account this model can be a very fast way to perform initial calculations to test if an EBG will have the desired effect inside the designed antenna structure.

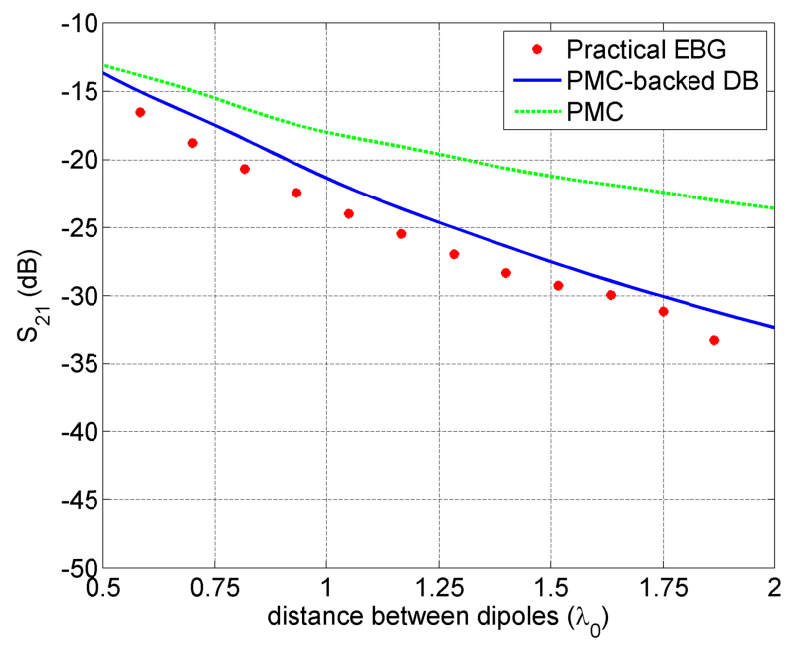

(a)

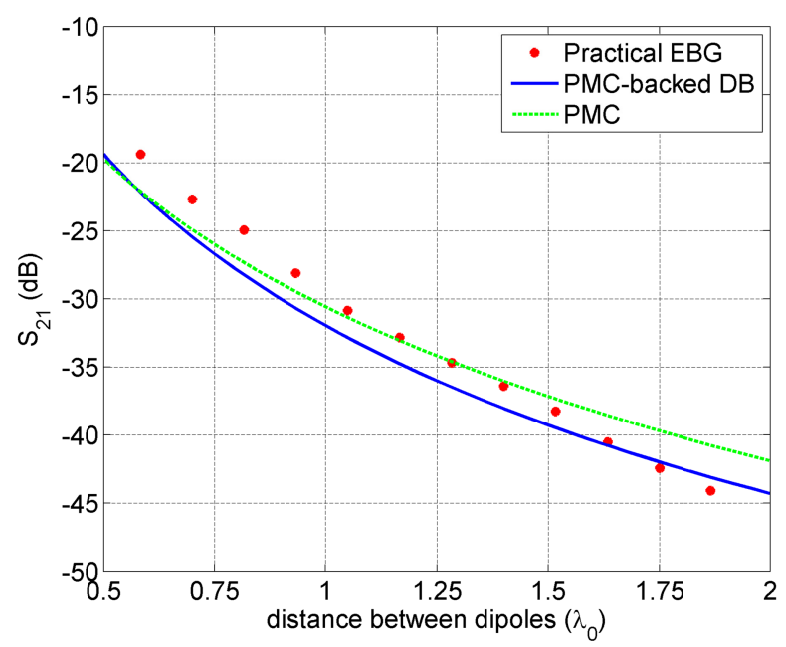

(b)

Fig. 7. Coupling between two dipoles above different structures (actual mushroom structure, ideal PMC and PMC-backed uniaxial model) versus their distance along the surface; (a) H-plane, (b) E-plane.

\section{ACKNOWLEDGMENT}

The authors would like to thank Prof. Stefano Maci from University of Siena, Italy and Prof. Ahmed A. Kishk from Concordia University, Canada for many fruitful discussions.

\section{REFERENCES}

[1] P.-S. Kildal, A. A. Kishk, and S. Maci, "Special issue on artificial magnetic conductors, soft/hard surfaces, and other complex surfaces" 
(Guest Editorial), IEEE Transactions on Antennas and Propagation, vol. 53, pp. 2-7, Jan. 2005.

[2] P.-S. Kildal and A. Kishk, "EM Modeling of surfaces with STOP or GO characteristics - artificial magnetic conductors and soft and hard surfaces," Applied Computational Electromagnetics Society Journal, Vol. 18, pp. 32-40, Mar. 2003.

[3] Fan Yang and Yahya Rahmat-Samii, Electromagnetic Band Gap Structures in Antenna Engineering, Cambridge University Press, 2009.

[4] P-S. Kildal, "Artificially soft and hard surfaces in electromagnetics," IEEE Trans. Antennas Propagat., Vol. 38, pp. 1537-1544, Oct. 1990.

[5] Ahmed Kishk and Per-Simon Kildal, "Modeling of soft and hard surfaces using ideal PEC/PMC strip grids," IET Microwaves, Antennas \& Propagation, Vol. 3, pp. 296-302, Mar. 2009.

[6] V. Lindell, and A.H. Sihvola, "Electromagnetic Boundary Conditions Defined in Terms of Normal Field Components," IEEE Trans. Antennas Propagat., Vol. 58, pp. 1128-1135, Apr. 2010.

[7] D. Sievenpiper, L.J. Zhang, R.F.J Broas, N.G.Alexopolous, E. Yablonovitch, "High-impedance electromagnetic surfaces with a forbidden frequency band," IEEE Transactions on Microwave Theory and Techniques, Vol. 47, No.11, pp. 2059-2074, November 1999.

[8] H. Rumsey, "Some new forms of Huygens' principle," IRE Trans. Antennas Propagat., Vol. 7, pp. 103-116, Dec. 1959.

[9] V. Lindell, A. H. Sihvola, "Electromagnetic boundary and its realization with anisotropic metamaterial," PHYSICAL REVIEW E 79, 026604, 2009.

[10] V. Lindell, and A.H. Sihvola, "Uniaxial IB-medium interface and novel boundary conditions," IEEE Transactions on Antennas and Propagation, vol. 57, pp. 694-700, Mar. 2009.

[11] P.-S. Kildal, A. Kishk, M. Bosiljevac, Z. Sipus, "The PMC-amended DB Boundary - A Canonical EBG Surface," Applied Computational Electromagnetics Society Journal, Vol. 26, pp. 96-108, Feb. 2011.

[12] D. M. Pozar, "Radiation and Scattering from a Microstrip Patch on a Uniaxial Substrate," IEEE Trans. Antennas Propagat., Vol. 35, pp. 613621, Jun. 1987.

[13] C. M. Krowne, "Green's Function in the Spectral Domain for Biaxial and Uniaxial Anisotropic Planar Dielectric Structures," IEEE Transactions on Antennas and Propagation, vol. 32, pp. 1273-1281, Dec. 1984.

[14] CST Microwave Studio 2014, www.cst.com.

[15] ADF-EMS, http://adf.ids-spa.it

[16] P. De Vita, F. De Vita, A. Di Maria, A. Freni, “An Efficient Technique for the Analysis of Large Multilayered Printed Arrays," IEEE Antennas and Wireless Propagation Letters, Vol. 8, pp. 104 - 107, 2009.

[17] D. M. Pozar, "Analysis of finite phased arrays of printed dipoles," IEEE Transactions on Antennas and Propagation, vol. 33, pp. 1045 - 1053, Oct. 1985. 